\title{
FORMULATION OF ANDROGRAPHOLIDE TRANSFERSOMES GEL FOR TRANSDERMAL DELIVERY: A PRELIMINARY STUDY
}

\author{
SILVIA SURINI*, PUTERI D. NASTITI, ADELIA R. PUTRI, KURNIA S. S. PUTRI
}

Laboratory of Pharmaceutics and Pharmaceutical Technology Development, Faculty of Pharmacy, Universitas Indonesia, Depok, West Java, Indonesia. Email: silvia@farmasi.ui.ac.id

Received: 26 September 2019, Revised and Accepted: 17 December 2019

\section{ABSTRACT}

Objective: A transfersome is a drug delivery system that offers increased penetration for the transdermal delivery of drugs. The aim of this study was to assess the application of transfersomes for transdermal delivery of andrographolide.

Methods: The development of andrographolide transfersomes for transdermal delivery was conducted in two steps. The first step involved varying ratios of Span 80 and phospholipids to investigate the effect on transfersome deformability using four formulations (P1-P4). Afterward, a second step involved varying ratios of andrographolide in transfersomes to investigate the influence on entrapment efficiency using four formulations (F1-F4). The selected transfersomes were then formulated into a gel dosage form. An in vitro penetration study was conducted by comparing the penetration fluxes of the transfersome and non-transfersome andrographolide gels using Franz diffusion cells.

Results: The results showed that formulation F4 had an entrapment efficiency of $97.02 \pm 0.01 \%$ and particle size $\left(\mathrm{D}_{\mathrm{v}{ }^{-} \text {average }}\right.$ ) of $524.02 \mathrm{~nm}$. An in vitro penetration study of the andrographolide transfersome gels showed two phases of penetration, with a marked increase in both fluxes. In the first phase, penetration flux was $23.26 \pm 2.34$ and $1.28 \pm 0.82 \mu \mathrm{g} / \mathrm{cm}^{2} \cdot \mathrm{h}$ for the transfersome and non-transfersome andrographolide gels, respectively.

Conclusion: The results showed that the transfersome gel is a promising dosage system for transdermal delivery of andrographolide.

Keywords: Andrographolide, Transfersome, Franz diffusion cell, Penetration study.

(c) 2020 The Authors. Published by Innovare Academic Sciences Pvt Ltd. This is an open access article under the CC BY license (http://creativecommons. org/licenses/by/4. 0/) DOI: http://dx.doi.org/10.22159/ijap.2020.v12s1.FF043

\section{INTRODUCTION}

Andrographolide is the main chemical constituent of the stem and leaves of Andrographis paniculata. The leaves of A. paniculata are traditionally orally consumed to relieve symptoms of the common cold, fever, and irregular bowel movement [1], and are also used as a patch to cover scars and prevent infection [1]. In addition, recent studies have reported the anticancer effects of andrographolide [2].

In a previous study, andrographolide was characterized as having a low absolute oral bioavailability of $2.67 \%$. Andrographolide is extensively metabolized in the duodenum and jejunum, and less so in the ileum and colon. The metabolism process results in an inactivated and sulfated form of andrographolide. High concentrations of sulfated andrographolide metabolites have been found in the liver, indicating extensive liver metabolism. At high doses, the oral bioavailability of andrographolide is hindered by its poor absorption in the terminal ileum and colon due to the presence of efflux transporters. About $5 \%$ of orally administered andrographolide is also excreted through bile, which further complicates the oral bioavailability of andrographolide. Taken together, oral administration of andrographolide at both low and high concentrations results in poor absolute oral bioavailability [3].

Transdermal delivery offers an alternative for the administration of andrographolide, which improves metabolism and absorption as compared with oral delivery. However, transdermal delivery may be hindered due to the low partition coefficient of andrographolide (log $p_{\text {app }}=0.59$ in the water at $\left.37^{\circ} \mathrm{C}\right)[4,5]$.

A transfersome is an artificial vesicle with the ability to change its shape and size to fit into much smaller spaces without rupturing to enhance the capability of an active ingredient to penetrate through the skin [6,7]. In recent studies, transfersomes were applied to enhance the delivery of large molecular or highly hydrophobic drugs across the skin. The aim of the present study was to investigate the application of transfersomes for the delivery of andrographolide.

\section{METHODS}

Materials

Andrographolide (98\%) was purchased from Xi'an Lyphar Biotech Co. Ltd. (Xi'an City, China). Phospholipon $90 \mathrm{G}$ was acquired from Lipoid GmbH (Ludwigshafen am Rhein, Germany). Sprague Dawley rats were obtained from Institut Pertanian Bogor (Bogor Agricultural University, Bogor, Indonesia). Other chemicals and solvents were of analytical grade and purchased from commercial suppliers.

Optimization and evaluation of andrographolide transfersomes An optimization study was conducted to select the most suitable ratio of phospholipid and Span 80 based on the deformability index. Andrographolide transfersome formulations P1-P4 were prepared with the use of the thin-film hydration method [8]. All transfersome formulations contained solid transfersomes at a concentration of $5 \mathrm{~g} / 100 \mathrm{~mL}$, with a ratio of andrographolide to phospholipid-Span 80 of 1:2 and a ratio of phospholipid to Span 80 of 95:5 (P1), 90:10 (P2), 85:15 (P3), or 80:20 (P4), as shown in Table 1.

Andrographolide, phospholipids, and Span 80 were dissolved in methanol by stirring at $200 \mathrm{rpm}$ for $20 \mathrm{~min}$. The solution was then transferred into a round-bottomed flask and evaporated using a rotary evaporator (Buchi model R-100; Buchi AG, Flawil, Switzerland) at $45 \pm 20^{\circ} \mathrm{C}$ under lowered pressure with stirring at $125 \mathrm{rpm}$. After all of the solvents were evaporated and a thin layer had formed on the walls of the flask, nitrogen gas was flowed into the flask for $2 \mathrm{~min}$. The flask was then refrigerated for $24 \mathrm{~h}$. Afterward, the thin layer was hydrated for $45 \mathrm{~min}$ with phosphate buffer (pH 7.4) at $40^{\circ} \mathrm{C}$ with constant stirring at $125 \mathrm{rpm}$. 
The deformability index was evaluated using the extrusion method [9]. Transfersomes were extruded using a Mini Extruder Kit (Avanti Polar Lipids Inc., Alabaster, AL, USA) through a membrane (pore size, $450 \mathrm{~nm}$ ) for $5 \mathrm{~min}$. The amount of transfersomes that were able to pass through the membrane was recorded and the particle size was measured. The deformability index was calculated with the use of the following formula:

$$
\mathrm{D}=\mathrm{J} \times\left(\frac{\mathrm{r}_{\mathrm{v}}}{\mathrm{r}_{\mathrm{p}}}\right)^{2}
$$

where, D=Deformability of the vesicle membrane, J=The amount of suspension extruded over a period of $5 \mathrm{~min}, \mathrm{r}_{\mathrm{v}}=$ Vesicle size after extrusion, and $r_{p}=$ Pore size of the barrier [10].

The particle size was also measured using a particle size analyzer (Malvern Zetasizer; Malvern Instruments, Malvern UK) in a suspension of one drop of transfersomes in $10 \mathrm{ml}$ of phosphate buffer ( $\mathrm{pH} 7.4)[7,11,12]$. The zeta potential was measured by testing one drop of transfersomes in $10 \mathrm{ml}$ of distilled water using the same equipment.

\section{Formulation and evaluation of andrographolide transfersomes}

Based on the results of the optimization study, four formulations were prepared by the thin-film hydration method [8]. All transfersome formulations contained solid transfersomes at a concentration of $5 \mathrm{~g} / 100 \mathrm{~mL}$, with a ratio of andrographolide to phospholipid-Span 80 of 1:2 (F1), 1:5 (F2), 1:10 (F3), or 1:15 (F4), as shown in Table 2 .

Andrographolide, phospholipids, and Span 80 were dissolved in methanol by stirring at $200 \mathrm{rpm}$ for $20 \mathrm{~min}$. The solution was then transferred into a round-bottomed flask and evaporated using a rotary evaporator (R-100) equipped with a vacuum pump (model V-100; Buchi AG) at $45 \pm 20^{\circ} \mathrm{C}$ at a lowered pressure with stirring at $125 \mathrm{rpm}$. After all of the solvents were evaporated and a thin layer had formed on the walls of the flask, nitrogen gas was flowed into the flask for $2 \mathrm{~min}$. The flask was then refrigerated for $24 \mathrm{~h}$. Afterward, the thin layer was hydrated for $45 \mathrm{~min}$ using phosphate buffer $\left(\mathrm{pH} \mathrm{7.4)}\right.$ at $40^{\circ} \mathrm{C}$ with constant stirring at $125 \mathrm{rpm}$.

Entrapment efficiency was evaluated using the direct method. Andrographolide transfersomes were separated from unentrapped andrographolide by centrifugation (CP100WX Ultracentrifuge; Hitachi High-Technologies Corporation, Tokyo, Japan) at 10,000 rpm for $60 \mathrm{~min}$. The precipitate was separated from the filtrate by decantation and washed 2 times with phosphate buffer ( $\mathrm{pH}$ 7.4). The precipitate containing andrographolide transfersomes was reconstituted with methanol and shaken until properly diluted. The resulting solution was

Table 1: Formula optimization of andrographolide transfersomes

\begin{tabular}{lllll}
\hline Materials & \multicolumn{3}{l}{ Ratio (g) } & \\
\cline { 2 - 5 } & P1 & P2 & P3 & P4 \\
\hline Andrographolide & 1.67 & 1.67 & 1.67 & 1.67 \\
Phospholipid & 3.17 & 3.00 & 2.83 & 2.67 \\
Span 80 & 0.17 & 0.33 & 0.50 & 0.67 \\
Phosphate buffer pH 7.4 & ad 100 & ad 100 & ad 100 & ad 100 \\
\hline
\end{tabular}

Table 2: Formulations of andrographolide transfersomes

\begin{tabular}{lllll}
\hline Materials & \multicolumn{3}{l}{ Ratio (g) } & \\
\cline { 2 - 5 } & F1 & F2 & F3 & F4 \\
\hline Andrographolide & 1.67 & 0.83 & 0.45 & 0.31 \\
Phospholipid & 2.67 & 3.33 & 3.64 & 3.75 \\
Span 80 & 0.67 & 0.83 & 0.91 & 0.94 \\
Phosphate buffer pH 7.4 & ad 100 & ad 100 & ad 100 & ad 100 \\
\hline
\end{tabular}

filtered through a filter with a pore size of $0.45 \mu \mathrm{m}$. Andrographolide in the transfersomes was quantified with the use of a high-performance liquid chromatography system [13,14] (model LC-20AT; Shimadzu Corporation, Kyoto, Japan) equipped with an ultraviolet (UV) absorbance detector (model SPD-20A; Shimadzu Corporation) at $223 \mathrm{~nm}$. A C18 column was used as the stationary phase, whereas the mobile phase was methanol-water at a ratio of 65:35. The flow rate for the analysis was $1.0 \mathrm{~mL} / \mathrm{min}$

The particle size in one drop of transfersomes in $10 \mathrm{ml}$ of phosphate buffer (pH 7.4) $[7,11,12]$ was measured using a particle size analyzer (Malvern Zetasizer). Transfersome morphology was evaluated using a transmission electron microscope (TEM) (200 kV FEI Tecnai F20 SuperTwin; Thermo Fisher Scientific, Waltham, MA, USA). The transfersome suspension was negatively stained by placing one drop on a carboncoated grid and adding one drop of $1 \%$ phosphotungstic acid. Any excess liquid was removed and the sample was examined using a TEM [11].

Formulation and evaluation of the andrographolide transfersome gel The gel base was formulated by thoroughly dispersing $750 \mathrm{mg}$ of carbomer (Carbopol ${ }^{\circledR}$ Ultrez 30 polymer; Lubrizol Corporation, Wickliffe, $\mathrm{OH}, \mathrm{USA}$ ) [12] in distilled water. Then, $500 \mathrm{mg}$ of triethanolamine was added until a mass had formed. Afterward, $12.5 \mathrm{~g}$ of propylene glycol was added, followed by $50 \mathrm{ml}$ of andrographolide transfersomes (equivalent to $150 \mathrm{mg}$ of andrographolide). A control gel was formulated by dissolving $150 \mathrm{mg}$ of andrographolide in a mixture of methanol and phosphate buffer (pH 7.4) into the gel base. Both andrographolide gels were evaluated for physical appearance, pH (H 510 microprocessor-based benchtop meter; Eutech Instruments Pte Ltd., Singapore), and viscosity (Brookfield viscometer; AMETEK Brookfield, Middleborough, MA, USA).

\section{In vitro penetration study}

In vitro penetration capabilities were examined using Franz diffusion cells (Mitra Cellular, Bali, Indonesia) [12]. The abdominal skin of rats (age, 8-10 weeks; weight, $\sim 200 \mathrm{~g}$ ) was used as a membrane for the penetration study. The methods used for animal sacrifice were approved by the Ethical Clearance Committee of Dr. Cipto Mangunkusumo Hospital, Faculty of Medicine, Universitas Indonesia (approval no. 0447/UN2.FI/ETIK/2018)

The Franz diffusion cell receptor compartment contained 10\% methanol solution in phosphate buffer ( $\mathrm{pH} 7.4)$ and was constantly stirred at $250 \mathrm{rpm}$ at $37 \pm 20^{\circ} \mathrm{C}$. Samples $(2 \mathrm{ml}$ ) were collected periodically over a 24-h period. The amount of penetrated andrographolide was quantified using a high-performance liquid chromatography system equipped with a UV absorbance detector at $223 \mathrm{~nm}$, as described above and elsewhere $[13,14]$. A C18 column was used as the stationary phase with a mobile phase of methanol-water at a ratio of 65:35. The flow rate for analysis was $1.0 \mathrm{ml} / \mathrm{min}$.

\section{RESULTS}

Optimization and evaluation of andrographolide transfersomes Transfersome formulations $\mathrm{P} 1-\mathrm{P} 4$ were opaque white in appearance and tended to settle on long-term storage. However, it was easy to re-disperse the sediments. The deformability study indicated that P4 had the highest deformability (Table 3). The particle sizes of the andrographolide transfersomes and the related zeta potentials are shown in Table 4. An increase in particle size was associated with an increase in the concentration of Span 80.

\section{Formulation and evaluation of andrographolide transfersomes} Transfersomes F1-F4 formed colloidal dispersions that were white in color. Similar to the optimized formulations, the transfersomes tended to settle upon long-term storage, but were able to be re-dispersed.

The entrapment efficiency and particle size of formulations F1-F4 are shown in Table 5. The entrapment efficiency was in the range of 46.62$97.02 \%$. Among the four formulations, F1 had the lowest entrapment efficiency and F4 had the highest. 
Table 3: Deformability index of the optimized andrographolide transfersome formulations

\begin{tabular}{|c|c|c|c|c|}
\hline Formula & $\begin{array}{l}\text { Amount of extruded } \\
\text { transfersomes }(\mathrm{mL})\end{array}$ & $\begin{array}{l}\text { Particle size before } \\
\text { extrusion }(\mathrm{nm})\end{array}$ & $\begin{array}{l}\text { Particle size } \\
\text { after extrusion (nm) }\end{array}$ & Deformability index \\
\hline P1 & 0.09 & 571.33 & 48.2 & 0.0010 \\
\hline P2 & 0.34 & 2098.73 & 50.9 & 0.0044 \\
\hline P3 & 0.19 & 612.70 & 57.9 & 0.0032 \\
\hline $\mathrm{P} 4$ & 0.45 & 625.00 & 136.9 & 0.0417 \\
\hline
\end{tabular}

Table 4: Particle size and zeta potential measurements of the optimized andrographolide transfersomes

\begin{tabular}{llllll}
\hline Formula & $\mathbf{D}_{\mathbf{v}-\mathbf{0}}(\mathbf{n m})$ & $\mathbf{D}_{\mathbf{v}-\mathbf{5 0}}(\mathbf{n m})$ & $\mathbf{D}_{\mathrm{v}-\mathbf{9} \mathbf{0}}(\mathbf{n m})$ & $\mathbf{D}_{\mathbf{v}-\text {-averge }}(\mathbf{n m})$ & Zeta Potential $(\mathbf{m V})$ \\
\hline P1 & 77.23 & 377.67 & 571.33 & 339.96 & -0.21 \\
P2 & 85.47 & 112.60 & 2098.73 & 5400.00 & -6.17 \\
P3 & 391.30 & 443.30 & 612.70 & 498.01 & -10.70 \\
P4 & 403.00 & 502.75 & 625.00 & 507.32 & -6.39 \\
\hline
\end{tabular}

Table 5: Measurements of particle sizes and entrapment efficiency of andrographolide transfersomes

\begin{tabular}{|c|c|c|c|c|c|c|}
\hline Formula & $D_{v-10}(n m)$ & $D_{v-50}(n m)$ & $D_{v-90}(n m)$ & $D_{\text {v-average }}(\mathrm{nm})$ & PDI & $\mathrm{EE}(\%)^{*}$ \\
\hline F1 & 616 & 825 & 1100 & 842.49 & 0.61 & $46,62 \pm 0,01$ \\
\hline $\mathrm{F} 2$ & 460 & 639 & 1910 & 845.29 & 0.86 & $55,77 \pm 0,01$ \\
\hline F3 & 524 & 762 & 5560 & 1941.58 & 0.95 & $59,16 \pm 0,03$ \\
\hline F4 & 405 & 516 & 668 & 524.02 & 1.00 & $97,02 \pm 0,01$ \\
\hline
\end{tabular}

*(mean \pm standard deviation, $\mathrm{n}=3$ ), PDI: Polydispersity index

Transfersome size was relatively large, with a high polydispersity index (PDI). The transfersome particle sizes and distribution patterns are shown in Fig. 1. Andrographolide transfersomes were spherical in shape, as shown in Fig. 2.

Formulation and evaluation of the andrographolide transfersome gel Gels containing andrographolide transfersomes were opaque white in color. The $\mathrm{pH}$ of the gels was rather acidic at 5.86 , and the viscosity was $60,000 \mathrm{cps}$. On the other hand, non-transfersome andrographolide gels were almost transparent in color with a slightly elevated $\mathrm{pH}$ of 6.38 and an elevated viscosity of $68,000 \mathrm{cps}$.

\section{In vitro penetration study}

Andrographolide transfersome gels showed two distinct phases of penetration. Gels containing andrographolide transfersomes showed a burst effect in an in vitro penetration study at $2.5 \mathrm{~min}$. The flux of the first phase was $23.258 \mu \mathrm{g} / \mathrm{cm}^{2} \cdot \mathrm{h}$ and lasted for $1 \mathrm{~h}$. The flux over the next hour had increased to $24 \mathrm{~h} 2.708 \mu \mathrm{g} / \mathrm{cm}^{2} \cdot \mathrm{h}$.

Comparatively, non-transfersome andrographolide gels also had two phases, an initial flux of $1.280 \mu \mathrm{g} / \mathrm{cm}^{2} \cdot \mathrm{h}$ for the first $3 \mathrm{~h}$ and a second flux of $0.327 \mu \mathrm{g} / \mathrm{cm}^{2} \cdot \mathrm{h}$ until $24 \mathrm{~h}$. The penetration profiles of both andrographolide gels are shown in Fig. 3 and the results of a comparison of the flux of both andrographolide gels are shown in Fig. 4.

\section{DISCUSSION}

Optimization and evaluation of andrographolide transfersomes Transfersomes were prepared by thin-film hydration, which is widely used in many studies, as this method is relatively easy to conduct in the laboratory setting $[11,12,15]$. Optimized formulations contained andrographolide and transfersomes at a 1:2 ratio. Formulations were prepared using the surfactant Span 80 because its hydrophobicity is pharmaceutically compatible with that of andrographolide, which could benefit entrapment efficiency [8].

A phosphate buffer at $\mathrm{pH} 7.4$ was chosen as the hydrating solution in this study to achieve favorable transdermal delivery of andrographolide. Phosphatidylcholine has an isoelectric point between 6 and 7. Due to the more basic nature of phosphate buffer, phosphatidylcholine has a net negative charge. The negative charge of transfersomes facilitates the transdermal delivery of andrographolide and other drugs [16].
Deformability index is an important characteristic of transfersomes reflecting the ability to transverse the stratum corneum and still retain the shape. The surfactant concentration in the transfersome formulations has been shown to affect the deformability of transfersomes by increasing plasticity [8]. Accordingly, the concentration of Span 80 in the transfersomes was chosen as a parameter in the optimization process. Formulations P1-P4 had increasing amounts of Span 80 in the phospholipid portion (5\%, 10\%, 15\%, and 20\%, respectively). The effect of the surfactant concentration on deformability is evidenced in the four formulation with F4 having the highest deformability and F1 having the lowest.

An increase in size was noted with an increase in the Span 80 concentration. An increase in the concentration of Span 80 in the formulations tended to increase the size of the transfersomes, as indicated in a study by Gupta et al. [7]. The zeta potential of all formulations was in the negative ranges, which was due to the net negative charge of phosphatidylcholine in phosphate buffer at pH 7.4 [17].

Formulation and evaluation of andrographolide transfersomes Andrographolide transfersomes were prepared by the thin-film hydration method. Formulations F1-F4 all contained 20\% Span 80 in the phospholipid portion. An increase in the phospholipid content in transfersomes affects the entrapment efficiency of andrographolide. Hence, this phenomenon was further explored $[6,8]$. Formulations F1-F4 contained andrographolide in decreasing ratios in respect to phospholipid-Span 80 (1:2, 1:5, 1:10, and 1:15, respectively). The obtained transfersomes were milky white in color.

The entrapment efficiency of formulations F1-F4 had increased with an increase in the lipid content. F4 had the highest phospholipidandrographolide ratio, which resulted in remarkable entrapment efficiency. The entrapment efficiency of transfersomes will increase as more phospholipids are added into the formulation. The addition of lipids increased the rigidity of the transfersomes, which improved the efficiency of andrographolide encapsulation [6].

Furthermore, the addition of $20 \%$ Span 80 to the formulations proved to be beneficial. For example, Gupta et al. reported a maximum entrapment efficiency in transfersome formulations containing $20 \%$ surfactant. A further increase in the surfactant content had no effect on the entrapment efficiency of transfersomes [7]. 


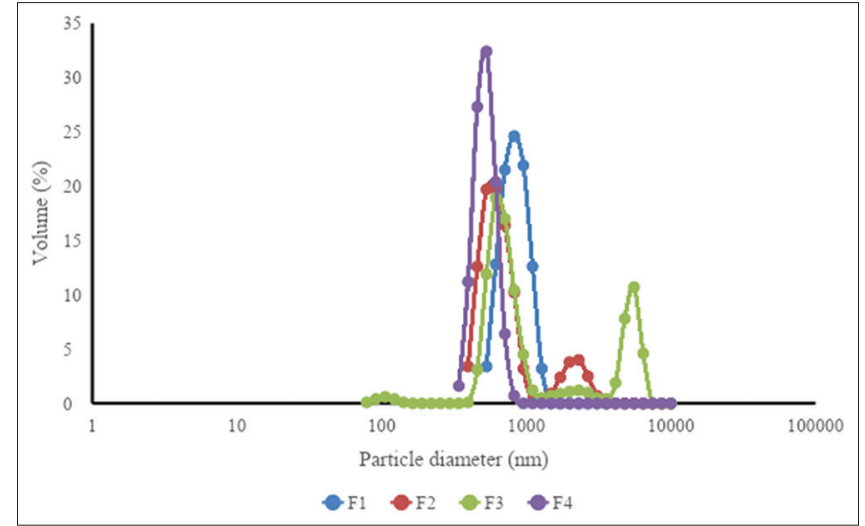

Fig. 1: Particle size distribution of andrographolide transfersomes (F1-F4)

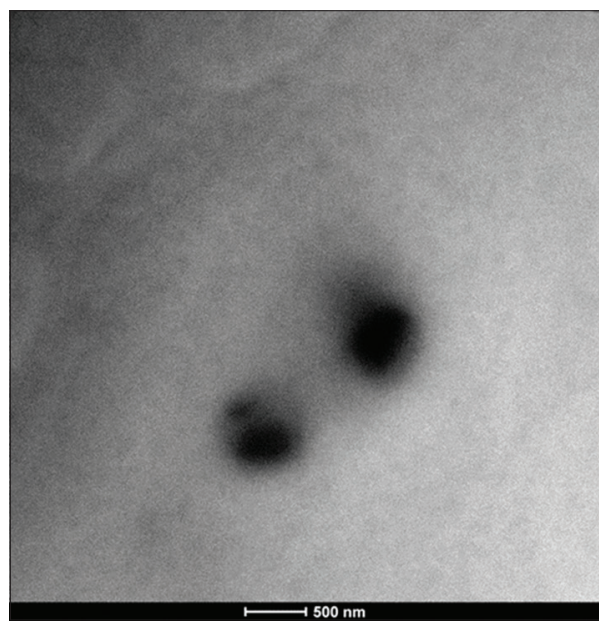

Fig. 2: Morphology representation of andrographolide transfersomes of F1

Transfersomes were large in size with a high polydispersity (PDI $=0.61-$ 1.00). The tendency of transfersomes to settle on long-term storage indicates a low zeta potential that could affect size stability, leading to the formation of larger vesicles by fusing with one another. This could explain the polydispersity that was observed in formulations F1-F4. As a preventive measure to hinder the fusion of vesicles, the transfersomes were stored at $4^{\circ} \mathrm{C}$ because the fusogenicity of transfersomes seems to be influenced by storage temperature, where a higher temperature (e.g., $37^{\circ} \mathrm{C}$ ) seems to increase the fusion of transfersomes [18].

The spherical morphology confirms the vesicular characteristics of andrographolide transfersomes. Morphologically, transfersomes do not tend to form multilamellar vesicles. The unilamellar spherical shape was formed due to the binding of the phosphatidylcholine heads of the molecules in the presence of water. This formation traps the hydrophobic andrographolide molecules in the lipophobic ends of phosphatidylcholine, thus encapsulating it and forming a vesicle.

Formulation and evaluation of the andrographolide transfersome gel The white color of the andrographolide transfersome gel can be attributed to the white color of the transfersomes. The viscosity of the andrographolide transfersome gel is lower than that of nontransfersome andrographolide gel because of the lower acidity. Viscosity is altered by the acidity of the gel because carbomer, the gelling agent of both andrographolide gels, favors a basic $\mathrm{pH}$ [19].

\section{In vitro penetration study}

The results of the in vitro penetration study showed that andrographolide was able to penetrate across the skin with or without

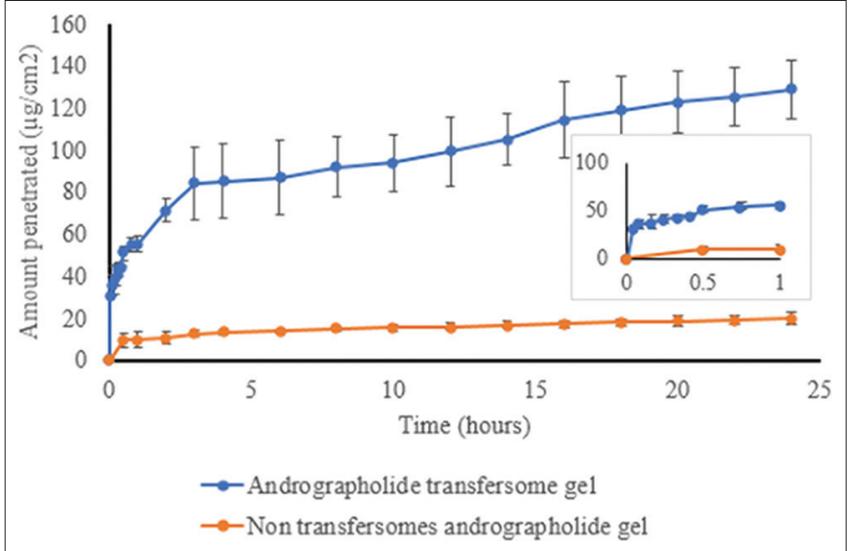

Fig. 3: Penetration profiles of andrographolide gels. Blue dots indicate andrographolide transfersome gel and orange dots indicate non-transfersome andrographolide gel

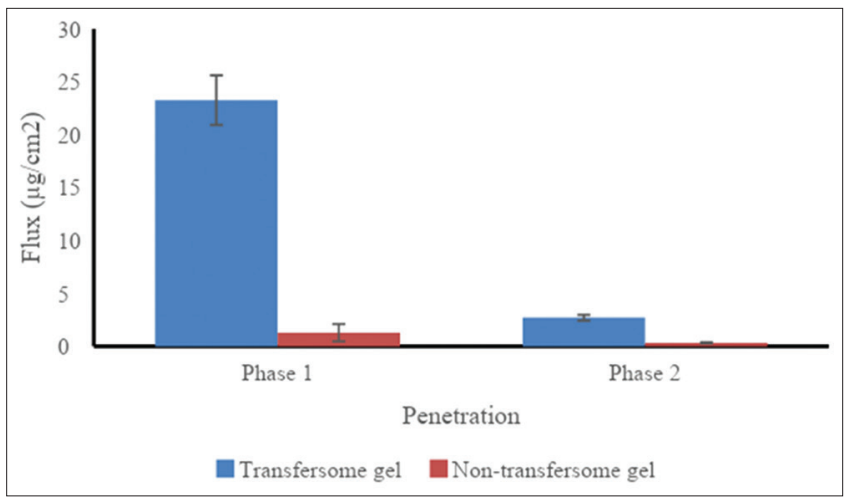

Fig. 4: Flux comparison of andrographolide gels. Blue bars indicate the flux of andrographolide transfersome gel and orange bars indicate the flux of non-transfersome andrographolide gel

transfersomes. However, there was a marked increase in penetration across skin with a gel containing andrographolide transfersomes, which was further evidenced by a marked increase in fluxes of penetration when compared to the control gel. These results show that encapsulation in transfersomes highly increased transdermal delivery of andrographolide.

The mechanism underlying transfersome transport of andrographolide through the skin can be explained by a difference in osmotic gradients between the stratum corneum (15\% water content) and epidermis (75\% water content). This difference provides a driving force for transfersomes to penetrate through the skin. This tendency is caused by innate characteristics of transfersomes to prevent dehydration and rupture. This transport is further assisted by deformability, which enables transport of transfersomes through the skin with minimal effects on the shape and size $[18,20]$.

The two distinct phases in the penetration of andrographolide gel may have been due to the deposition of andrographolide on rat skin. Initially, andrographolide can rapidly penetrate through the skin, which indicates a release of a surface-absorbed drug. Afterward, andrographolide, both as free drug and as a transfersome, is deposited in the subcutaneous tissue, which acts as a reservoir that provides a slower, delayed drug release, thereby explaining the distinct penetration phase [18]. Further studies of andrographolide skin deposition are needed to confirm this hypothesis.

Another mechanism that could explain the two-phase penetration profile of andrographolide transfersome gel is the deceleration caused 
by the heterogeneity of transfersome sizes. Due to the low deformability of andrographolide transfersomes, larger transfersome vesicles cannot cross the skin. It is also likely that the transport of larger transfersomes is hindered due to the physical characteristics of the vesicles [20].

\section{CONCLUSION}

Andrographolide transfersome formulation $\mathrm{F} 4$ resulted in vesicles with an entrapment efficiency of $97.02 \pm 0.01 \%$ with $\mathrm{D}_{\mathrm{v} \text {-average }}$ of $524.02 \mathrm{~nm}$. The gel containing andrographolide transfersomes revealed a marked increase in penetration capability in vitro, as compared to the nontransfersome andrographolide gel, suggesting that the andrographolide transfersome gel is a promising potential system for transdermal delivery of andrographolide. In addition, it is recommended that andrographolide transfersomes with more homogenous particle sizes and better deformability should be developed to ensure successful transdermal delivery of andrographolide.

\section{ACKNOWLEDGMENTS}

The authors gratefully acknowledge Universitas Indonesia for support and a PITTA research grant 2019.

\section{CONFLICTS OF INTEREST}

The authors declare that they have no conflicts of interest.

\section{REFERENCES}

1. Akbar S. Andrographis paniculata: A review of pharmacological activities and clinical effects. Altern Med Rev 2011;16:66-77.

2. Banerjee M, Chattopadhyay S, Choudhuri T, Bera R, Kumar S, Chakraborty B, et al. Cytotoxicity and cell cycle arrest induced by andrographolide lead to programmed cell death of MDA-MB-231 breast cancer cell line. J Biomed Sci 2016;23:40.

3. Ye L, Wang T, Tang L, Liu W, Yang Z, Zhou J, et al. Poor oral bioavailability of a promising anticancer agent andrographolide is due to extensive metabolism and efflux by P-glycoprotein. J Pharm Sci 2011;100:5007-17.

4. Korinth G, Wellner T, Schaller KH, Drexler H. Potential of the octanolwater partition coefficient $(\log \mathrm{P})$ to predict the dermal penetration behaviour of amphiphilic compounds in aqueous solutions. Toxicol Lett 2012;215:49-53.

5. Yao Y, Liao QF, Zeng LY, Zhang L, Ma Y, Zeng YE. Determination of apparent oil-water partition coefficient of andrographolide and dehydroandrographolide and effect of $\mathrm{pH}$ on them. Zhong Yao Cai 2009;32:1610-2.
6. Chaudhary H, Kohli K, Kumar V. Nano-transfersomes as a novel carrier for transdermal delivery. Int J Pharm 2013;454:367-80.

7. Gupta A, Aggarwal G, Singla S, Arora R. Transfersomes: A novel vesicular carrier for enhanced transdermal delivery of sertraline: Development, characterization, and performance evaluation. Sci Pharm 2012;80:1061-80.

8. Qushawy M, Nasr A, Abd-Alhaseeb M, Swidan S. Design, optimization and characterization of a transfersomal gel using miconazole nitrate for the treatment of Candida skin infections. Pharmaceutics 2018;10:e26.

9. Luthfiah A, Sagita E, Iskandarsyah I. Physical stability testing of p-synephrine prepared as transfersome gel. Int J Appl Pharm 2017;9:124-6.

10. van den Bergh BA, Wertz PW, Junginger HE, Bouwstra JA. Elasticity of vesicles assessed by electron spin resonance, electron microscopy and extrusion measurements. Int J Pharm 2001;217:13-24.

11. El Zaafarany GM, Awad GA, Holayel SM, Mortada ND. Role of edge activators and surface charge in developing ultradeformable vesicles with enhanced skin delivery. Int J Pharm 2010;397:164-72.

12. Surini S, Sarah S, Djajadisastra J. Formulation and in vitro penetration study of transfersomes gel containing gotu kola leaves extract (Centella asiatica L. Urban). J Young Pharm 2017:9:483-6.

13. Sajeeb BK, Kumar U, Halder S, Bachar SC. Identification and quantification of andrographolide from Andrographis paniculata (Burm. f.) wall. ex nees by RP-HPLC method and standardization of its market preparations. J Pharm Sci 2015;14:71-8.

14. Sinha J, Mukhopadhyay S, Das N, Basu MK. Targeting of liposomal andrographolide to L. donovani infected macrophages in vivo. Drug Deliv 2000; 7:209-13.

15. Setyawati DR, Surini S, Mardliyati E. Optimization of luteolin-loaded transfersome using response surface methodology. Int J Appl Pharm 2017;9:107-11

16. Sinico C, Manconi M, Peppi M, Lai F, Valenti D, Fadda AM. Liposomes as carriers for dermal delivery of tretinoin: In vitro evaluation of drug permeation and vesicle-skin interaction. J Control Release 2005;103:123-36.

17. Duangjit S, Opanasopit $\mathrm{P}$, Rojanarata $\mathrm{T}$, Ngawhirunpat $\mathrm{T}$. Characterization and in vitro skin permeation of meloxicam-loaded liposomes versus transfersomes. J Drug Deliv 2011;2011:418316.

18. Jain S, Jain P, Umamaheshwari RB, Jain NK. Transfersomes--a novel vesicular carrier for enhanced transdermal delivery: Development, characterization, and performance evaluation. Drug Dev Ind Pharm 2003;29:1013-26.

19. Rowe R, Sheskey P, Quinn M, editors. Handbook of Pharmaceutical Excipients. Vol. 6. London: Pharmaceutical Press; 2009.

20. Rajan R, Jose S, Mukund VP, Vasudevan DT. Transferosomes a vesicular transdermal delivery system for enhanced drug permeation. J Adv Pharm Technol Res 2011;2:138-43. 